\title{
How should we evaluate non-pharmacological treatments in child and adolescent psychiatry?
}

\author{
Bruno Falissard ${ }^{1}$
}

Published online: 21 August 2015

(C) Springer-Verlag Berlin Heidelberg 2015

\section{Introduction}

Non-pharmacological treatments are crucial for child and adolescent psychiatry. Despite many available therapies, only a minority of them have been well evaluated. This month there were two papers published in the Journal that evaluated such treatments. This is very encouraging. The study designs used in both cases were randomized controlled trials (RCTs), the gold standard from a methodological point of view. But is it reasonable to require such a demanding methodological approach to evaluation in every instance? Are there not other alternatives to evaluation? Here are some proposed reflections on the issue to launch an open forum discussion, which may likely go beyond the question of evaluation.

Proposition 1 The success of occidental medicine has been obtained at the cost of a marginalization of the mind and of a physiological authoritarianism.

Following Comte's epistemology, medicine has evolved over the centuries from a theological stage (shamanism) to a metaphysical stage (Hippocrates' theory of humors) and ultimately, to a positive stage emerging with Andreas Vesalius, which reached its apogee with Claude Bernard in the XIXth century. The amazing impact of anatomy and physiology in the progression of therapeutics has subsequently shaped occidental medicine. Doctors are now trained in specializations according to organs (cardiology, urology,

Bruno Falissard

bruno.falissard@gmail.com

1 Université Paris-Sud, INSERM U1178, Maison de Solenn, 97 Bd de Port Royal, 75679 Paris Cedex, France pneumology, etc.) and medical studies rely heavily on biology courses. As a consequence, we have lost a global medical approach that holistically incorporated, culture and mind, mind and body, and body and organs. The impact of this loss has been particularly important in disciplines such as child and adolescent psychiatry, where culture and mind play an important role.

Proposition 2 Physiology is only part of the equation, and it cannot be used as the ultimate gold standard to evaluate treatments.

Physiologists have long had the idea that deterministic models can comprehensively describe human functioning and can be used to assess the evaluation of treatment. This was made explicit in a well-known text of Claude Bernard [1]:

"In the patient, who succumbed, the cause of death was evidently something, which was not found in the patient who recovered; this something we must determine, and then we can act on the phenomena or recognize or foresee them accurately; then only scientific determinism will be achieved. But not by statistics shall we succeed in this; never have statistics taught anything, and never can they teach anything about the nature of phenomena. I shall apply what I have just said to all the statistics compiled with the object of learning the efficacy of certain remedies in curing diseases."

Unfortunately this idea never came to reality and consequently is the reason why RCTs appeared in the middle of the XXth century [2]. Randomized controlled trials can be considered as a probabilistic equivalent to the experimental method of physiologists. But there is an important 
difference to observe: there is no universal law in the efficacy of treatments, merely statistical laws that exist.

More generally, even in this XXIth century, there is still no comprehensive theory that can describe a patient (i.e., a subject with organs and cells, who thinks and who lives in a society). Biology is thus only a part of the equation.

Proposition 3 Randomized controlled trials follow a tradition deeply rooted in the biological paradigm, and thus, they are not neutral.

Even if some of the physicians who promoted RCTs questioned very early the relevance of a pure biological paradigm in medicine [2], RCTs are de facto dependent on this paradigm in many aspects. The prominent role of the placebo in the evaluation of medical treatment is a good illustration of this. For example, a comparison of medication versus a placebo allows us to say that a treatment has a specific effect, i.e., an effect of its chemical composition. Curiously, RCTs are not carried out specifically to evaluate if treatments are beneficial in day-to-day clinical practice. This is because RCTs do not assess a benefit/risk ratio but rather, they mainly assess benefits while ignoring completely (at least on a statistical point of view) the balance between benefit and risk. Further, due to the question of blinding, it is generally impossible to compare a treatment to the "best standard of care," which in many cases would be the most relevant alternative for practitioners. Randomized control trials (RCTs) are in fact a form of protection against pseudoscience and irrationality, and this is precisely why the placebo is so important. Of course, medicine and patients alike have suffered a lot from pseudoscience over the centuries and protection from this has become of major importance. But the problem is elsewhere. To prove that a treatment has efficacy, RCTs and modern standards of evaluation are posed with the task of demonstrating that the particular treatment in question is better than the placebo, i.e., that the treatment has a specific effect according to the biological paradigm. This is, however, an abuse of dominant position. Because there is currently no grand unified theory of what a patient is, there is no reason that a unique theory be binding to prove that a treatment is effective. Concerning the evaluation of nonpharmacological treatment in psychiatry, this point is crucial because most of these treatments are developed outside the biological paradigm. This is one of the reasons why in many cases it is absurd to ask for a comparison of the placebo in the evaluation of a psychological treatment.

Proposition 4 From a methodological point of view, RCTs are only a partial success.

The RCT is indeed an interesting design. In short, the $R C T$ is the most efficient design in providing a convincing answer to a simple question (i.e., "is treatment 'A' better than treatment ' $\mathrm{B}$ ' on a given outcome measure?"). The RCT is however no more than that. In particular, it is improper to affirm that RCTs can prove any causal relationship between a treatment and its effects. In a particular sense of causality (counterfactual causality), if a trial were perfectly organized, one might be able to claim existence of a causal association but only in probability, only for the average patient. But because RCTs are never perfectly organized, and because an "average patient" does not exist, in practice, the affirmation of causality is excessive, if not fallacious [5].

RCTs have other weaknesses.

First, RCTs do not answer the question: "How do patients feel once undergoing treatment?" Take the case of depression-a RCT will prove that patients improve more with treatment 'A' than 'B' based on a score from a particular scale. Patients are then on average "less depressed." But does this mean that patients indeed feel "better"? A qualitative study was recently conducted with patients treated by SSRIs using an interpretative phenomenological analysis approach [4]. An emotional disconnection was found with experiences like: "I could 'hear' the anxiety yelling somewhere inside me, but it was muffled, as if someone had put up soundproof glass." Or "It's easier to encounter all this dark stuff, and not feel too- that boundaries are-not get sucked into it. It was cool, it was like, 'wooh! All this stuff is dark and scary, but it's not going to get me!" This type of information regarding treatment effects as seen from the patient's point of view is very valuable for clinical practice, and yet is not provided by RCTs.

In addition, RCTs are not optimal for determining which patients will be good or bad responders, for detecting rare and/or severe side effects, for assessing a cost efficiency ratio or even for assessing benefits within a real-life setting.

\section{Conclusion}

For centuries, patients have been proposed treatments that were quackery. This danger is still present and we have to carefully evaluate the therapies that we propose, whether they are pharmacological or not. Science is obviously the cornerstone of evaluation for medicine in general and for psychiatry in particular. But today, medical scientific thought is stationary because of two erroneous and deleterious postulates. The first, biology is the only theoretical framework relevant in medicine and psychiatry. Second, quantitative approaches (particularly randomized designs) are the only standards for evaluating treatments.

Because there is presently no "grand unified theory" of what a patient is, theories coming from the social and human sciences should be considered just as legitimate as 
those coming from biology within the field of medicine in general and for treatment evaluation in particular. Even if the RCT is a very interesting and powerful design, RCTs have many inherent drawbacks and thus cannot be considered as the philosophical cornerstone for the evaluation of all therapies. The two erroneous postulates outlined above have been deleterious because they have led us to absurd situations. For instance, if a new treatment is able to optimize some so-called non-specific aspects of healing, this treatment may be considered as a placebo and thus as a quackery. This is not acceptable because such treatments may in fact be useful for our patients and because it is likely that many non-pharmacological treatments fall into this situation.

Finally, how should we evaluate non-pharmacological treatments in child and adolescent psychiatry?

There are a wide range of tools that can be used and many of them should be systematically considered. First, a precise description of the treatment under evaluation should be systematically provided. The biological or chemical formula of a new medication is essential; but the process of a new psychological therapy is just as essential. The mechanism of action of such new treatments should also be studied, and this is in line with some papers which recently created a stir [3]. Of course, if the therapy under study is not developed according to a biological perspective, there is no need to look for a biological mechanism of action; other theories are welcome. Qualitative studies can be used to finely characterize the effects of treatment on the patient's existence. RCTs are then best placed to answer the question: "Is the treatment better than a given alternative?" Remember that the concept of placebo is not likely to be relevant when a non-pharmacological treatment is considered. Non-randomized cohorts will be able to look for good responders, for possible side effects and even for health results within an economic framework. Of course, it is of major importance to carefully and systematically identify possible side effects whether treatments are pharmacological or not.

Ideally, all these studies should be done simultaneously because they are complementary in nature. In practice, monetary resources will impact upon, and constrain the evaluation methods that we are able to carry out when considering new treatments. Regrettably, the amount of money invested in the evaluation of non-pharmacological treatments is so insignificant compared to what is invested towards the evaluation of medications. From both a clinical and public health perspective, this latter point lacks rational justification.

Acknowledgments The author warmly thanks Lisa Christine Girard for her help in the English editing of the paper.

\section{References}

1. Bernard C (1957) An introduction to the study of experimental medicine. Dover Publications, New York

2. Marks HM (2000) The progress of experiment: science and therapeutic reform in the United States, 1900-1990, 1st edn. Cambridge University Press, Cambridge

3. Reardon S (2014) NIH rethinks psychiatry trials. Nature 507(7492):288. doi: $10.1038 / 507288 \mathrm{a}$

4. Teal J (2009) Nothing personal: an empirical phenomenological study of the experience of being-on-an-SSRI. J Phenomenol Psychol 40(1):19-50. doi:10.1163/156916209X427972

5. Worrall J (2007) Why there's no cause to randomize. Br J Philos Sci 58(3):451-488. doi:10.1093/bjps/axm024 